\title{
Über das Finden von Grauen Schwänen
}

\author{
Effektives Risiko-Management sollte neben quantifizierbaren, bekannten Risiken auch \\ Ereignisse berücksichtigen, die entweder in ähnlicher Art bereits eingetreten oder \\ grundsätzlich vorstellbar sind. Für eine Identifikation dieser "Grauen Schwäne“ müssen \\ institutionell-organisatorische Voraussetzungen geschaffen und analytisch-konzeptionelle \\ Instrumente bereitgestellt werden.
}

Julia Brüggemann, Christoph Binder

War die COVID-19-Pandemie unvorhersehbar? - Fakt ist: Viele Unternehmen konnten sich eine Krise dieses Ausmaßes nicht vorstellen und waren nur unzureichend vorbereitet. Nicht so die US-amerikanische Supermarktkette H-E-B mit 370 Filialen in Texas und Nordmexiko. Bereits 2005, nach dem Ausbruch der Vogelgrippe (H5N1), hatte H-E-B Pandemien als generelles Risiko für ihr Geschäftsmodell identifiziert und einen Notfallplan entwickelt. Diesen wendete sie 2009 beim Ausbruch der Schweinegrippe (N1N1) an. Basierend auf den Erfahrungen reagierte die Supermarktkette bereits Mitte Januar 2020. Die ergriffenen Maßnahmen umfassten Befragungen von Zulieferern in China über die Auswirkungen vor Ort auf Lieferketten, Mitarbeiterverfügbarkeit, Einkaufsverhalten, Versorgung bei Quarantäne et cetera. Die gewonnenen Erkenntnisse wandte $\mathrm{H}$-E-B modellhaft auf ihre Verkaufsregionen an. Damit war es möglich, die COVID19-Auswirkungen deutlich zu reduzieren (vergleiche Solomon/Forbes 2020).

\section{Schwarze und Graue Schwäne}

In der öffentlichen Diskussion wird COVID-19 häufig als „Schwarzer Schwan“ bezeichnet. Dieser von Nassim Nicolas
Taleb geprägte Begriff beschreibt äußerst seltene Ereignisse mit erheblichen - meist negativen - Auswirkungen, die jeweils bis zu ihrem Eintreten nicht vorhergesehen wurden (vergleiche Taleb 2007, S. xvii-xviii). Solche Ereignisse werden häufig auch als „unknown unknowns“ bezeichnet, also Sachverhalte, „Von denen wir nicht wissen, dass wir sie nicht wissen“ (Rumsfeld 2002, o. S.). Da solche Events mit gängigen wissenschaftlichen Methoden nicht erfassbar sind, ist es laut Taleb auch nicht zielführend zu versuchen, sie im Voraus zu identifizieren. Stattdessen ist ein Management der Auswirkungen von Schwarzen Schwänen empfehlenswert (vergleiche Taleb/Goldstein/Spitznagel 2009, S. 78), etwa über den Aufbau von Resilienz, Agilität sowie ein gutes Krisen-Management (vergleiche Schäffer 2020).

Andere vertreten dagegen die Meinung, dass eine „Demaskierung“ (de-blankening) von Schwarzen Schwänen durch den Austausch von Wissen beziehungsweise das Auswerten von Daten möglich ist (vergleiche beispielsweise Lindaas/ Petersen 2016, S. 1236; Batrouni/Bertaux/Nicolle 2018). Eine solche Demaskierung von Schwarzen Schwänen zu „Grauen Schwänen“ hält auch Taleb selbst für grundsätzlich realisierbar (vergleiche Taleb 2007, S. 213 ff.). 
Graue Schwäne, auch als „known unknowns“ bezeichnet, werden dabei als eine Art Fenster in die Zukunft verstanden. Es sind Ereignisse, die entweder in ähnlicher Art bereits eingetreten sind, etwa ein Tsunami, oder die man sich als grundsätzlich möglich vorstellen kann. Denkbar wäre beispielsweise ein Cyber-Angriff auf die deutsche Stromversorgung mit daraus folgenden mehrtägigen Stromausfällen. Die Auswirkungen eines Grauen Schwans können erheblich sein, sie sind allerdings meist ungewiss.

Ob ein Event als Schwarzer oder Grauer Schwan einzustufen ist, hängt letztlich vom Betrachter, seiner Situation und ganz besonders seinem aktuellen Wissensstand ab (vergleiche Aven 2013, S. 49). Berücksichtigt man die Vorgeschichte, so spricht vieles dafür, die COVID-19-Pandemie in die Kategorie Grauer Schwan einzuordnen: Die Ausbrüche von Vogel- und Schweinegrippe 2005 beziehungsweise 2009 wiesen auf eine eindeutige Bedrohungslage hin. Auch Bill Gates warnte 2015 öffentlich vor einer globalen Pandemie. Zudem wurde bereits 2011 eine Modellierung der wirtschaftlichen Effekte einer Grippe-Pandemie veröffentlicht (vergleiche Verikios et al. 2011).

Für viele Unternehmen kam COVID-19 trotzdem völlig überraschend. Ihr Fokus lag vielfach vor allem auf quantifizierbaren, bekannten Risiken („known knowns") ebenso wie auf der Erfüllung rechtlicher Vorschriften im Rahmen des Risiko-Managements. Solch ein Ansatz ist jedoch unvollständig, will ein Unternehmen sich auch auf echte oder vermeintliche Graue Schwäne vorbereiten (vergleiche Kaplan/Leonard/ Mikes 2020, S. 11 f.). Um problem- und situationsgerechter mit ihnen umgehen zu können, muss das Unternehmen institutionell-organisatorische Voraussetzungen schaffen sowie geeignete analytisch-konzeptionelle Instrumente bereitstellen.

\section{Institutionell-organisatorische Voraussetzungen}

Angesichts zunehmend kürzerer Verweildauern im Amt sowie einer Fülle von Kontroll- und Haftungsrisiken mag es für manche Vorstands- oder Aufsichtsratsmitglieder durchaus verlockend erscheinen, über die Demaskierung von Schwarzen Schwänen nicht allzu genau Bescheid zu wissen. Denn aus derartigem Wissen erwächst die Notwendigkeit, die entsprechenden Maßnahmen einzuleiten, geeignete Organisationsund Führungsstrukturen zu schaffen und auch die Kontrollaufgaben kontinuierlich wahrzunehmen. Dies ist erfahrungsgemäß nicht immer einfach.

Um den langfristigen Erhalt und die Wettbewerbsfähigkeit des Unternehmens zu sichern, ist eine Beschäftigung mit Grauen Schwänen aber unabdinglich. Voraussetzung ist eine

\section{Zusammenfassung}

- Unternehmen können sich auf viele Ereignisse, die auf den ersten Blick unvorhersehbar scheinen, vorbereiten. Die Beschäftigung mit diesen „Grauen Schwänen“ trägt dazu bei, den langfristigen Erhalt und die Wettbewerbsfähigkeit von Unternehmen zu sichern.

- Voraussetzungen für eine erfolgreiche Identifikation von Grauen Schwänen sind eine positive Risikokultur sowie eine hohe Wertigkeit des Risiko-Managements auf Vorstands- und Aufsichtsratsebene.

- Durch eine systematische Nutzung von qualitativen und quantitativen Instrumenten, integriert in den strategischen Planungsprozess, können konventionelle Denkweisen und überkommene Lösungsansätze hinterfragt und damit die Resilienz des Unternehmens gestärkt werden.

positive Risikokultur im Unternehmen. Hierzu muss die Vorstands- und Aufsichtsratsebene der Identifikation und dem Management von Risiken im Allgemeinen und Grauen Schwänen im Speziellen eine hohe Wichtigkeit einräumen. Bedrohungen und Ängste müssen identifiziert und eskaliert werden dürfen. Führungskräfte müssen dies entsprechend vorleben und Bonussysteme, Beförderungskriterien sowie die

\section{„Wie die COVID-19-Pandemie verdeut- licht, ist die Beschäftigung mit Grauen Schwänen von hoher Relevanz."}

Corporate Governance darauf angepasst sein. Ein positives Beispiel hierfür ist eine europäische Großbank, die die klare Maßgabe an die Geschäftseinheiten herausgegeben hat, dass Verluste akzeptiert werden, solange die entsprechenden Risiken vorher identifiziert wurden. Mit negativen Konsequenzen ist nur zu rechnen, wenn Risiken offensichtlich nicht adressiert wurden.

\section{Analytisch-konzeptionelle Instrumente}

Um das Problem von nicht oder kaum vorhersehbaren Ereignissen zu adressieren, bieten sich aus heutiger Sicht vor allem zwei Typen von Instrumenten an: (1) Qualitative sowie quantitative Instrumente, wie sie aus dem Enterprise Risk Manage- 
ment (ERM) bekannt sind; (2) Szenariobasierte Ansätze, wie sie inzwischen im Rahmen einer weiterentwickelten strategischen Planung Eingang gefunden haben (vergleiche Abbildung 1).

\section{Qualitative ERM-Instrumente}

Diese Instrumente haben eindeutig explorativen Charakter. Ziel ist, mit ihrer Hilfe jeweils für das Unternehmen relevante Graue Schwäne zu identifizieren beziehungsweise Schwarze Schwäne zu demaskieren. Bereits vorhandene Informationen, Wissen sowie Einschätzungen werden dazu unternehmensintern, gegebenenfalls auch mit externer Unterstützung durch hinzugezogene Experten, systematisch gesammelt und gesichtet. Methodisch am geläufigsten sind BrainstormingAnwendungen, da sie relativ einfach und kostengünstig realisierbar sind. In aller Regel entwickelt hierbei eine Gruppe von Personen zu einer vorher definierten Problemstellung in einer begrenzten Zeit möglichst viele Ideen. Sie wird dabei üblicherweise von einem Experten in der Moderatorenrolle unterstützt. Unternehmen nutzen Brainstormings oft, um gravierende, atypische Geschäftsrisiken zu erkennen. In einer in- ternationalen Investment-Bank beispielsweise ist es üblich, zweimal im Jahr Brainstormings auf Top-Management-Ebene abzuhalten, um die Eintrittswahrscheinlichkeiten und finanziellen Auswirkungen von „known unknowns“ zu diskutieren. Als Diskussionsbasis dient ein vorbereiteter Katalog mit rund 50 Events unterschiedlichster Art - von Pandemien, geomagnetischen Sonnenstürmen bis zur plötzlichen Verstaatlichung von Auslandsgesellschaften, etwa in China.

Wesentlicher Nachteil von Brainstormings ist die Anfälligkeit für kognitive Verzerrungen (Biases), die aus einer systematisch fehlerhaften - meist unterbewussten - Voreingenommenheit bei Denkprozessen, Meinungsbildungen, Entscheidungen, aber auch ganz allgemein in der menschlichen Interaktion resultieren. Solche Verhaltensmuster wurden unter anderem von den Psychologen Kahneman und Tversky beschrieben und auch in Experimenten bestätigt (vergleiche Kahneman/Tversky 1979; Kahneman 2011). Besonders auffällige Erscheinungsformen im Rahmen des Brainstormings sind das sogenannte Group Thinking, der Authority Bias und der Confirmation Bias: Typisch für Gruppendenken ist die

Abb. 1 Analytisch-konzeptionelle Instrumente zur Risiko-Identifikation von Grauen Schwänen

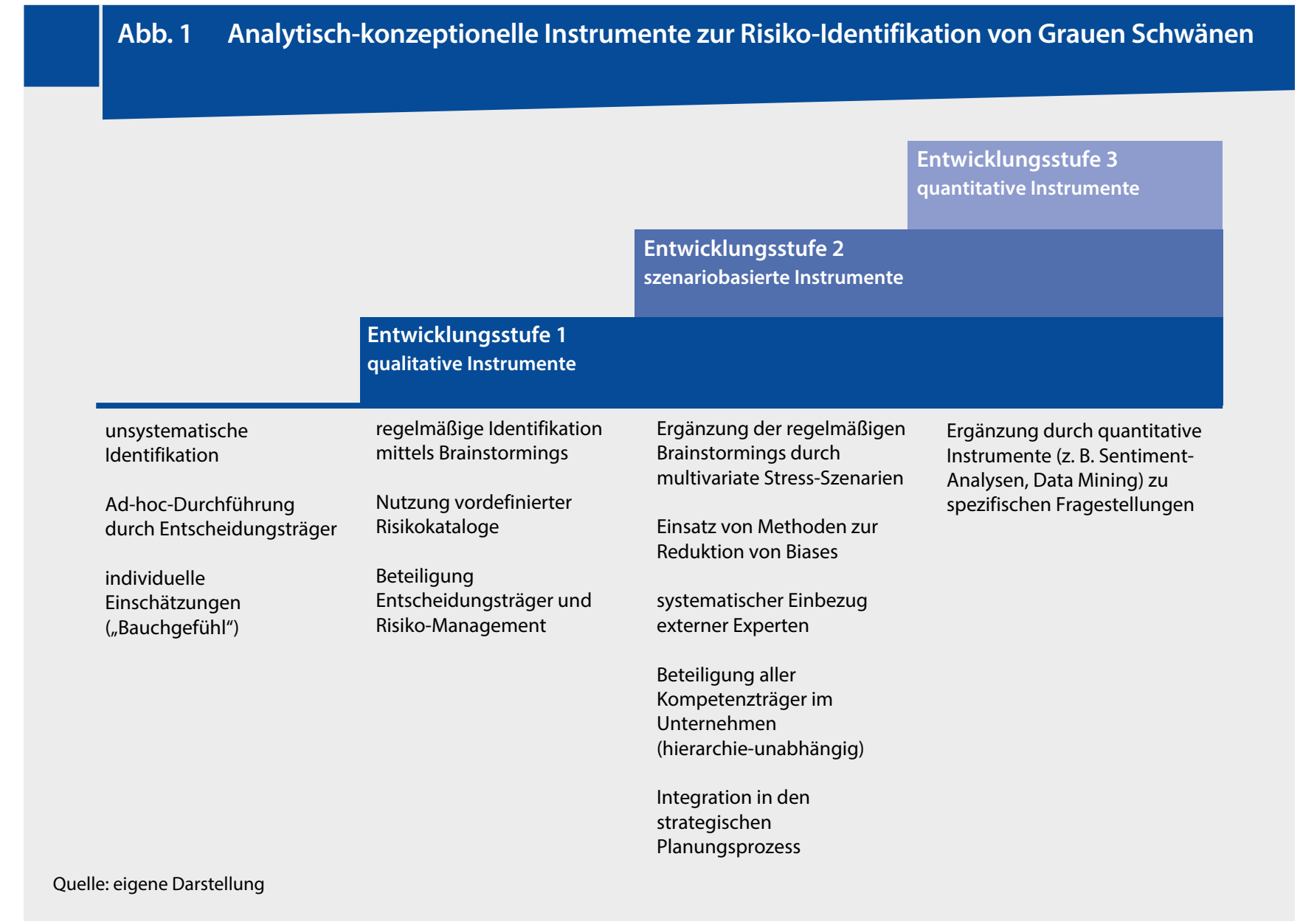


Bemühung um Harmonie und Konsens innerhalb einer Gruppe mit der Folge, dass erreichte Diskussionsstände gegebenenfalls nicht (mehr) infrage gestellt und alternative Ansätze nicht (mehr) erwogen werden. Autoritäten - Experten, aber auch hierarchisch höherstehenden Personen - wird zudem häufig von vornherein ein höheres Maß an Sachkenntnis zugebilligt als etwa Durchschnittsmenschen. Daneben tendieren Personen mit ausgeprägten Voreinstellungen dazu, Informationen so zu interpretieren, dass sie jeweils die eigenen Erwartungen bestätigen.

Gerade solche Verzerrungen führen dazu, dass mittels Brainstormings eher selten neue Graue Schwäne identifiziert werden können. Eine Reihe instrumenteller Weiterentwicklungen des Brainstormings zielt darauf ab, mögliche Biases durch prozessuale Anpassungen sowie organisatorische Änderungen zu reduzieren sowie die Kreativität und Vorstellungskraft der Teilnehmer verstärkt anzuregen. Am bedeutsamsten sind die nachstehenden Ansätze.

Premortem-Technik: Statt zu fragen „Was könnte künftig passieren?", wird hier bereits ein konkretes negatives Ereignis angenommen, beispielsweise das deutliche Verfehlen des Geschäftsergebnisses. Aufgabe der Brainstorming-Teilnehmer ist es, Gründe für dieses Ergebnis zu finden und in einer Diskussion zu plausibilisieren (vergleiche Klein 2007, S. 1). Ziel dieser Technik ist es, Barrieren zu überwinden, sodass potenzielle Probleme - etwa bei starkem Hierarchiegefälle innerhalb der Gruppe - leichter zur Sprache gebracht werden können. Auf Teilnehmerseite erhält man so ein breiteres Spektrum an Sichtweisen - und als Beiprodukt Hinweise auf ähnlich gelagerte, ebenfalls nur schwer vorhersehbare potenzielle Gefährdungen (vergleiche Gallop/Willy/Bischoff 2016, S. 104). Denkbare Ursachen wie auch mögliche Wirkungsketten können besser identifiziert werden.

Red Teaming: Bei dieser Methode haben sogenannte „Red Teams", wie sie seit Langem aus militärischen Planspielen bekannt sind, die explizite Aufgabe, alternative Ereignis-Interpretationen vorzuschlagen und etabliertes Denken infrage zu stellen. Einzelpersonen oder Teams übernehmen die Rolle eines konkreten Wettbewerbers oder gar Gegners und treten als Advocatus Diaboli auf. Ziel ist, das Blickfeld auf potenzielle Probleme zu erweitern und Graue Schwäne besser zu identifizieren (vergleiche Masys 2012, S. 323).

Einbeziehung externer Experten: Gerade für Themenfelder, in denen Unternehmen erfahrungsgemäß nur über wenig eigene Expertise verfügen, ist es ratsam, externe Experten hinzuzuziehen. Solche Themenfelder sind etwa Geopolitik, technologischer Wandel, globaler Klima- und Artenschutz et cetera. So hat es sich ein amerikanischer Energiekonzern zum Anliegen gemacht, regelmäßig ehemalige Botschafter zum Brainstorming über geopolitische Risiken einzuladen.

Anpassungen beim Ablauf: Um einer Verengung von Perspektiven und Ansätzen entgegenzuwirken, kann es sinnvoll sein, die Teilnehmer noch vor Brainstorming-Beginn ihre Meinungen aufschreiben zu lassen (vergleiche Lovallo/Sibony 2010). Ebenso sollte die Identifikation von möglichen „known unknowns" klar getrennt werden von der Diskussion über Eintrittswahrscheinlichkeiten oder mögliche Risikoumfänge. Denn nur so lässt sich ein strikter Fokus auf Graue Schwäne aufrechterhalten.

\section{"Zu erwarten ist, dass sich insbesondere KI-basierte Analysetechniken über die Zeit immer besser zur Identifizierung von Grauen Schwänen nutzen lassen werden."}

Neben diesen vier Ansätzen ist auf die Diversität der Teilnehmer zu achten, um den Zugang zu einem breiteren Spektrum an Wissen und Erfahrungen sicherzustellen. Zudem ist das richtige Umfeld wichtig. Bewährt hat sich, Brainstormings bewusst abseits des Geschäftsbetriebs durchzuführen, ohne den üblichen Arbeits- und Zeitdruck, gegebenenfalls mit Unterstützung durch externe Experten und Moderatoren.

\section{Quantitative ERM-Instrumente}

Unternehmen stehen heute enorme, ständig wachsende Mengen an internen und externen Daten zur Verfügung, beispielsweise Tweets und Blog-Einträge, Google Trends oder Fehlerraten in Produktionsprozessen. Diese lassen sich mit modernen Analysetechniken zunehmend produktiver nutzen. So hilft etwa Data Mining, statistische Gesetzmäßigkeiten oder Muster systematisch aufzuspüren. Machine Learning kann eingesetzt werden, um erkannte Zusammenhänge auch auf neue beziehungsweise andere Datensätze zu übertragen.

$\mathrm{Zu}$ erwarten ist, dass sich quantitative, insbesondere KIbasierte Analysetechniken über die Zeit immer besser zur Identifizierung von Grauen Schwänen nutzen lassen werden. Erste Beispiele für fokussierte Fragestellungen gibt es bereits: „Licensee Event Reports“ werden dazu genutzt, organisatorische Risikofaktoren in der US-amerikanischen Atomindustrie zu identifizieren (vergleiche Pence et al. 2020). Der „Thomson 
Reuters Clear Risk Inform“ dient dazu, die Risiken ziviler Unruhen zu erfassen (vergleiche Leidner 2015, S. 11) und die kanadische Firma Aquantix nutzt KI, um basierend auf Satellitendaten, Wetterprognosen und sonstigen öffentlichen Dokumenten den Wasserbedarf von Unternehmen sowie die Wahrscheinlichkeit von Wasserrisiken (Überschwemmungen, Dürren) in einer Region abzuschätzen (vergleiche The Economist 2021, S. 60).

\section{„Es ist unumgänglich, sich in gewissem Umfang auch neue Expertise für die Planungs- und Risiko-Management- Abteilungen aufzubauen."}

Die Beispiele zeigen, dass quantitative Methoden zur Identifizierung von Grauen Schwänen derzeit noch eher experimentell eingesetzt werden. Hemmnisse sind hohe Kosten und ein im Zweifelsfall unklarer Wertsteigerungsbeitrag sowie das bislang nur begrenzte Know-how und die knappen Personalund Sachressourcen für umfassende quantitative Datenanalysen. Über kurz oder lang ist gleichwohl zu erwarten, dass sich die neuen Methoden und Technologien der quantitativen Datenanalyse immer stärker durchsetzen und wie selbstverständlich zur Identifizierung von Grauen Schwänen genutzt werden. In anderen Unternehmensbereichen bereits aufgebaute Analyse-Expertise kann dabei einen Best-PracticeTransfer ermöglichen.

\section{Szenariobasierte Ansätze in der strategischen Planung}

Vor allem Unternehmen der Öl- und Gasindustrie, aber auch Rüstungsfirmen und Start-ups haben bereits Szenarioanalysen zur Identifizierung von Grauen Schwänen in den strategischen Planungsprozess integriert. Ihr Ziel ist, damit neue Trends sowie potenzielle Störfaktoren für die zukünftige Entwicklung der strategischen Geschäftsfelder zu identifizieren und zu quantifizieren. Diese werden dann in den Planungsrunden vertiefend diskutiert. Häufig orientieren sich die Szenarien aber an der Gegenwart („expected case“), mit nur relativ leichten Abweichungen nach oben und unten („best and worst case“). Diese „Linearisierung“ von Zukunftserwartungen hat zur Folge, dass „die Enden der Verteilungen“ abgeschnitten und Stress-Szenarien nicht entsprechend berücksichtigt werden (vergleiche Heiligtag/Maurenbrecher/Niemann 2017, S. 1). Ein gutes Beispiel hierfür sind die Szenarioanalysen zur Entwicklung des deut- schen Strompreises: Ausgehend von einem Großhandelspreis für eine Megawattstunde von knapp über 60 Euro im Jahre 2008, lag die Industrieerwartung im Niedrigpreisszenario bei 51 Euro für das Jahr 2016. Als Folge des Atomkraftunfalls in Fukushima 2011 und der damit einhergehenden Beschleunigung der Energiewende betrug der Preis 2016 tatsächlich lediglich etwa 22 Euro (vergleiche Heiligtag/Maurenbrecher/ Niemann 2017, S. 2).

Besondere Bedeutung im Rahmen szenariobasierter Ansätze haben multivariate Stress-Szenarien, die mehrere Risikofaktoren betrachten. Diese sollten zudem nicht nur die Effekte erster Ordnung, sondern auch mögliche Wirkungsketten und deren Auswirkungen auf die wesentlichen Planungsgrößen berücksichtigen. Bei der Erstellung dieser Stress-Szenarien kann durchaus auf die genannten qualitativen ERMInstrumente zurückgegriffen werden, um „known unknowns“ zu identifizieren. Die Definition der Szenarien sollte stets durch Personen erfolgen, die holistisch, integrativ (vergleiche Werther 2017, S. 358) und interdisziplinär denken und umfassende Industrieerfahrung mitbringen. Sie sollten insbesondere imstande sein, nicht nur den Status quo kritisch zu hinterfragen, sondern auch charakteristische Entwicklungsmuster frühzeitig zu erkennen. Solche Persönlichkeitsprofile finden sich indes nur selten in den traditionellen Planungsund Risiko-Management-Abteilungen. Damit ist es unumgänglich, sich in gewissem Umfang auch die entsprechende neue Expertise aufzubauen.

\section{Schlussbetrachtung}

Wie die COVID-19-Pandemie verdeutlicht, ist die Beschäftigung mit Grauen Schwänen für Unternehmen grundsätzlich von hoher Relevanz. Durch die Etablierung und fortlaufende Weiterentwicklung eines systematischen Vorgehens - sei es im Rahmen des ERMs oder durch die Integration in den strategischen Planungsprozess - können Graue Schwäne frühzeitig erkannt und damit negative Auswirkungen reduziert werden. Selbst wenn ein spezifischer Grauer Schwan nicht erkannt oder die Auswirkungen unterschätzt werden, so wird doch durch die regelmäßige Beschäftigung mit „known unknowns“ die Resilienz des Unternehmens gegen Krisen verbessert.

Die Identifikation von Grauen Schwänen lässt sich jedoch nur dann zielführend und erfolgreich implementieren, wenn damit auch eine Veränderung in der Denkweise der Beteiligten einhergeht. Die Beschäftigung mit Extremszenarien erlaubt es, in den Unternehmen konventionelle Denkweisen und überkommene Lösungsansätze einfacher und kritischer 
zu hinterfragen. Diese Chance zur Stärkung der institutionellen Resilienz im Unternehmen sollte unbedingt genutzt werden. Eine Integration in die strategische Planung verbessert zudem die Akzeptanz für den mit der Identifizierung von Grauen Schwänen verbundenen Arbeits- und Zeitaufwand. Ebenso gewinnt die Beschäftigung mit diesen Fragestellungen durch die mit der strategischen Planung einhergehende Quantifizierung der Auswirkungen an Stellenwert.

\section{Literatur}

Aven, T. (2013): On the meaning of a black swan in a risk context, in: Safety Science, 57, S. 44-51.

Batrouni, M./Bertaux, A./Nicolle, C. (2018): Scenario analysis, from Big Data to black swan, in: Computer Science Review, 28, S. 131-139.

The Economist (2021): Divining water risk - an expanding pool, 9. Januar, S. 60.

Gallop, D./Willy, C./Bischoff, J. (2016): How to catch a black swan: measuring the benefits of the pre-mortem technique for risk identification, in: Journal of Enterprise Transformation, 6 (2), S. 87-106.

Heiligtag, S./Maurenbrecher, S./Niemann, N. (2017): Applying stress tests beyond banking, in McKinsey Quarterly, Dezember.

Kahneman, D. (2011): Thinking, fast and slow, New York.

Kahneman, D./Tversky, A. (1979): Prospect theory: an analysis of decision under risk, in: Econometrica, 47 (2), S. 263-291.

Kaplan, R./Leonard, H./Mikes, A. (2020): Novel risks, Harvard Business School Working Paper, No. 20-094, 1, https://www.hbs.edu/ faculty/Pages/item.aspx?num=57892 (letzter Abruf: 10.12.2020).

Klein, G. (2007): Performing a project premortem, in: Harvard Business Review, September, S.18-19.

Leidner, N. (2015): Computer-supported risk identification for the holistic management of risks, Cornell University Working Paper, https://arxiv.org/pdf/1510.08285.pdf (letzter Abruf: 10.12.2020).

Lindaas, O./Petersen, K. (2016): Risk analysis and black swans: two strategies for de-blackening, in: Journal of Risk Research, 19 (10), S. 1231-1245.

Lovallo, N./Sibony, N. (2010): Taking the bias out of meetings, in: McKinsey Quarterly, April.

Masys, A. (2012): Black swans to grey swans: revealing the uncertainty, in: Disaster Prevention and Management, 21 (3), S. 320-335.

Pence, J./Farshadmanesh, P./Kim, J./Blake, C./Mohaghegh, Z. (2020): Data-theoretic approach for socio-technical risk analysis: test mining licensee event reports of U.S. nuclear power plants, in: Safety Science, 124 (April), S. 1-21.

Rumsfeld, D. (2002): News briefing by US Secretary of Defence, NATO Headquarter, 6. Juni, https://www.nato.int/docu/speech/2002/ s020606g.htm (letzter Abruf: 10.12.2020).

Schäffer, U. (2020): Levers of organizational resilience, in: Controlling \& Management Review, 64 (6-7), S. 8-19.

www.springerprofessional.de/link/18410504
Solomon, D./Forbes, P. (2020): Inside the story of how H-E-B planned for the pandemic, Texas Monthly, 26.03.2020, https://tinyurl. com/heb-coronavirus-pandemic (letzter Abruf: 10.12.2020).

Taleb. N. N. (2007): The black swan: the impact of the highly improbable, London.

Taleb, N. N./Golstein, D./Spitznagel, M. (2009): The six mistakes executives make in risk management, in: Harvard Business Review, 87 (October), S. 78-81.

Verikios, G./Sullivan, M./Stojanovski, P./Giesecke, J./Woo, G. (2011): The global economic effects of pandemic influenza, Conference Paper, https://static.rms.com/email/documents/liferisks/papers/ the-global-economic-effects-of-pandemic-influenza.pdf (letzter Abruf: 10.12.2020).

Werther, G. (2017): Improving finance and risk management foresight abilities: growing past the "black swan" mindset through integrative assessment, in: Journal of Risk Management in Financial Institutions, 10 (4), S. 353-364.

\section{Angaben zu den Autoren}

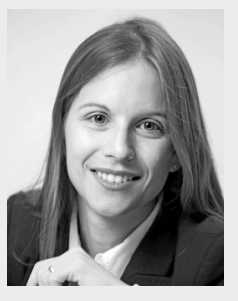

Prof. Dr. Julia Brüggemann

ist Professorin für Corporate Finance und

Risiko-Management an der ESB Business

School der Hochschule Reutlingen.

E-Mail: julia.brueggemann@reutlingen-

university.de

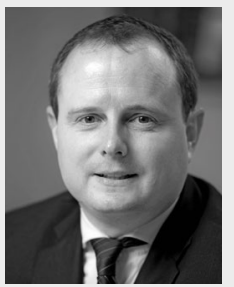

Prof. Dr. Christoph Binder

ist Professor für Management Accounting \& Controlling an der ESB Business School der Hochschule Reutlingen.

E-Mail: christoph.binder@reutlingenuniversity.de

\section{SpringerProfessional}

black swans

Brown, D. J. (2020): Affective decision making under uncertainty risk, ambiguity and black swans, Cham. www.springerprofessional.de/link/18700608

Zito, R. R. (2020): Bounding the "black swan" probability, in: Zito, R. R: Mathematical foundations of system safety engineering, Cham, S. 51-62.

www.springerprofessional.de/link/17379336

Park, Y. W./Hong, P. (2019): Paradigm shift and black swan strategy, in: Park, Y. W./Hong, P.: Creative innovative firms from Japan, Singapore, S. 41-50.

www.springerprofessional.de/link/17703990 